Dirk Fox

\title{
Elektronische Wahlgeräte
}

\section{Hintergrund}

Schon seit den 1960er Jahren wird in Deutschland über die Nutzung von Wahlgeräten zur Beschleunigung der Stimmauszählung bei Kommunal-, Landtagsund Bundestagswahlen nachgedacht. Das Bundeswahlgesetz (BWG) vom 07.05.1956 sah daher in $\$ 35$ die Nutzung von ,amtlich zugelassenen Stimmenzählgeräten“ vor, die vom Bundesministerium des Inneren (BMI) zu genehmigen waren [1].

Tatsächlich gab es Versuche mit mechanischen und elektromechanischen Zählwerken, die auf der Grundlage der „Verordnung über die Verwendung von Stimmenzählgeräten“vom 24.08.1961 und der späteren Bundeswahlgeräteverordnung (BWahlGV) vom 03.09.1975 zugelassen wurden, bei denen jedoch der für Beschaffung, Transport und Wartung erforderliche Aufwand den Nutzen bei weitem überwog. Einige Lösungen eigneten sich zudem nicht für den Einsatz mit langen Kandidatenlisten. $\mathrm{Zu}$ einem flächendeckenden Einsatz solcher „Stimmenzählgeräten" kam es daher nie.

Die Zuständigkeit für die Prüfung der Eignung von Wahlgeräten (Baumusterprüfung) - als Voraussetzung für eine Zulassung durch das BMI - wurde in der BWahlGV in die Hände der Physikalisch-Technischen Bundesanstalt in Braunschweig (PTB) gelegt ( $\$ 2$ Abs. 2 BWahlGV) [2].

\section{Elektronische Wahlgeräte}

Mit einem Antrag der niederländischen Firma Nederlandsche Apparatenfabriek (Nedap) auf Bauartzulassung der in den Niederlanden bereits intensiv genutzten Nedap-Wahlcomputer kam im Jahr 1997 Bewegung in das Thema. Mit einem softwaregesteuerten Wahlgerät, so die Befürworter, bestand die Chance, die Auszählung einer Wahl zu beschleunigen, ohne die „Bedienungsfreundlichkeit“ des Wahlvorgangs zu verringern. Auch die unbeabsichtigte Abgabe ungültiger Stimmen (nicht eindeutiges Kreuz o.ä.) sollte so ausgeschlossen werden.

Schließlich konnten durch den Einsatz von Wahlcomputern die Zahl der erforderlichen Stimmbezirke und die der be- nötigten Wahlhelfer nachweislich um 30$50 \%$ verringert werden [6].

Um den Einsatz der Nedap-Wahlcomputer bei der Europawahl im Juni 1999 in Deutschland zu ermöglichen, wurden aus $\S 35$ Abs. 1 BWG („Zur Erleichterung der Abgabe und Zählung der Stimmen können anstelle von Stimmzetteln Wahlumschlägen und Wahlurnen Wahlgeräte mit selbständigen Zählwerken benutzt werden.“) die Worte „mit selbständigen Zählwerken" gestrichen.

Am 20.04.1999 erließ das BMI eine Neufassung der BWahlGV, die in Anlage 1 „Richtlinien für die Bauart von Wahlgeräten" enthielt. Diese orientierten sich im Wesentlichen an den spezifischen Eigenschaften der Nedap-Geräte, wie z. B. die Anforderung, dass auf der Vorderseite des Wahlgeräts für jeden Wahlvorschlag „ein abgegrenztes Feld mit eindeutig zugeordneter Bedienungsvorrichtung zur Auswahl der Stimmabgabe "vorhanden sein muss [2] - eine Anforderung, die beispielsweise ein digitaler Wahlstift ${ }^{1}$ entwurfsbedingt nicht erfüllen kann.

Auf derselben rechtlichen Grundlage erhielten für die Bundestagswahl $2002 \mathrm{Ne}$ dap-Geräte vom Typ ESD-1 eine Bauartzulassung und wurden von 29 Kommunen in ihren Stimmbezirken eingesetzt. Bei der Bundestagswahl 2005 stieg die Zahl der eingesetzten Nedap-Geräte (Typ ESD-1 und ESD-2) auf über 1.800 Exemplare [5].

Die Baumusterprüfung durch die PTB umfasste inzwischen auch eine Softwareprüfung mit Hilfe von Codeinspektionen, dynamischen Funktionstests und einem Review der Entwicklungs- und Textdokumentation [3] in einem 2001 von der Deutschen Akkreditierungsstelle Technik (DATech) akkreditierten Prüflabor [4].

\section{Öffentlichkeit der Wahl}

Mit der am 03.03.2009 veröffentlichten Entscheidung erklärte der zweite Senat des Bundesverfassungsgerichts die BWahlGV für verfassungswidrig, weil sie keine dem Verfassungsgrundsatz der „Öffentlichkeit der Wahl“ (Art. 38 GG) entsprechende Kontrolle sicherstellt. Der Einsatz von

\footnotetext{
1 Siehe Maseberg, in diesem Heft.
}

Abb. 1 | Softwaregesteuertes Wahlgerät ESD1 der Firma Nedap; Quelle: www.ptb.de

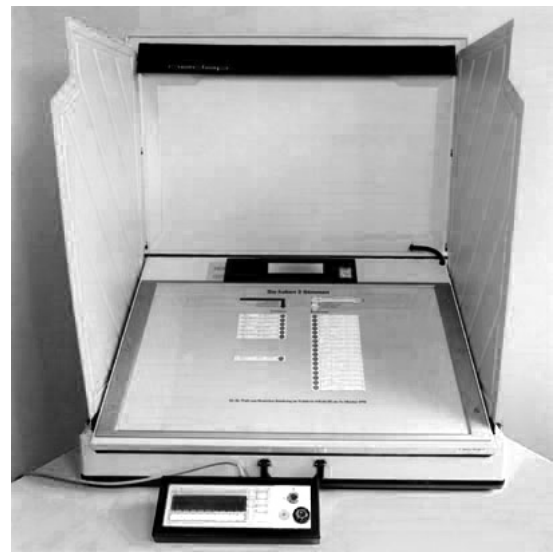

Wahlcomputern der Firma Nedap ist ebenfalls mit diesem Grundsatz unvereinbar [1].

Der Einsatz elektronischer Wahlgeräte kann nur unter sehr engen Voraussetzungen zulässig sein, da „die große Breitenwirkung möglicher Fehler an den Wahlgeräten oder gezielter Wahlfälschungen“ besondere Vorkehrungen zur Wahrung des Grundsatzes der Öffentlichkeit der Wahl fordert: Die „wesentlichen Schritte von Wahlhandlung und Ergebnisermittlung“ müssen „zuverlässig und ohne besondere Sachkenntnis überprüft werden können“" [1]. Diesem Erfordernis müssen die BWahlGV und die Eignungsprüfung genügen.

Referenzen

[1] Bundesverfassungsgericht, 2 BvC 3/07 vom 3.3.2009 http://www.bverfg.de/entscheidungen/cs20090303_2bvc000307.html

[2] Bundeswahlgeräteverordnung http://bundesrecht.juris.de/bwahlgv/index.html

[3] Physikalisch-technische Bundesanstalt (PTB): Elektronische Wahlgeräte, Arbeitsgruppe 8.5.1 vom 05.12.2006 http://www.berlin.ptb. de/8/85/851/Wahlgeraete.htm

[4] Physikalisch-technische Bundesanstalt (PTB): Softwareprüfstelle der PTB akkreditiert. http://www.ptb.de/de/publikationen/jahresberichte/jb2001/nachrdjahres/ndj09d.html

[5] Sietmann, Richard: Dreimal drücken - fertig?, c't 19/2005, S. 45 http://www.heise.de/ ct/05/19/054/

[6] Sietmann, Richard: Obskure Demokratie-Maschinen, c't 20/2006, S. 86-93. 